\title{
Redesign of a rural emergency department to prepare for the COVID-19 pandemic
}

\author{
Christopher Patey MD, Shabnam Asghari MD PhD, Paul Norman RN, Oliver Hurley MEnvSc
}

— Cite as: CMAJ 2020 May 11;192:E518-20. doi: 10.1503/cmaj.200509; early-released April 21, 2020

CMAJ Podcasts: author interview at https://soundcloud.com/cmajpodcasts/200509-com

I $\mathrm{n}$ the face of the current global pandemic, World Health Organization, regional and Canadian guidance reports have been published to assist health facilities to triage and manage the treatment of patients with suspected severe acute respiratory syndrome coronavirus 2 (SARS-CoV-2) infection..$^{1-3}$ Screeningg and triage may occur before admission to a hospital or community clinic or an emergency department. Current Canadian guidance does not provide specific, practical advice for rural emergency department administrators and staff on how to rapidly prepare for a potential surge in patients with coronavirus disease 2019 (COVID-19) in a way that will promote staff well-being, conserve personal protective equipment (PPE), protect patients presenting with non-COVID emergencies and mobilize external supports. ${ }^{3}$ Rural hospitals have limited staff and equipment resources, may be last to receive allocated supplies and often find it challenging to implement rapidly evolving, one-size-fits-all guidance on controlling local community and hospital transmission of a novel pathogen. Although guidance may suggest the transfer of severely ill patients from rural hospitals to tertiary referral centres, immediate transfer of severely ill patients with COVID-19 may be challenging in the Canadian setting. This means that rural hospital emergency departments should be prepared to manage a broad spectrum of patients presenting with COVID-19. We discuss our approach to preparing the emergency department of the rural hospital in Carbonear, NL, to face the COVID-19 pandemic, as a potential model for others to follow.

The hospital in Carbonear is an 80-bed hospital that provides emergency and inpatient services to a catchment population of about 40000 . The Carbonear emergency department has 8 stretchers and 3 high-turnover examination beds, and an average daily volume of about 80 patients. ${ }^{4}$ The emergency department team is made up of 1 primary family physician who practises emergency medicine, 1 secondary coverage physician, 1 nurse practitioner and up to 4 registered nurses per shift.

Appendix 1 (available at www.cmaj.ca/lookup/suppl/ doi:10.1503/cmaj.200509/-/DC1) describes the components of the preparedness and response plan of the Carbonear emergency department, which broadly involved engaging all emergency department staff in the plan; considering the patient perspective;

\section{KEY POINTS}

- Rapid preparation for a surge in patient flow in rural emergency departments during the coronavirus disease 2019 (COVID-19) pandemic is essential; however, current Canadian guidance does not provide specific, practical advice for rural emergency department administrators and staff on how to prepare.

- Our approach to preparing the emergency department of the rural hospital in Carbonear, NL, to face the COVID-19 pandemic may serve as a model for others to follow.

- Our pandemic response planning was characterized by close collaboration among administrators, the site clinical chief and front-line staff as well as optimization of communication channels.

- Front-line emergency department staff are physically, mentally and socially affected during a pandemic; our response plan considered available resources to support front-line staff.

- Owing to limited supplies of personal protective equipment (PPE), we reconfigured operations so as to optimize PPE usage.

restructuring the emergency department to allow for effective isolation of patients with potential COVID-19 from other patients and staff; finding ways to support front-line staff in terms of managing workload and mental well-being; reviewing safety; considering the potential effects of health worker infection and reduced workforce owing to self-isolation; and creating a forecasting model to anticipate surge.

The primary goal of COVID-19 emergency department operations planning was to decrease the risk of transmission and reasonably maintain the flow of patients through the department.

We developed a team of administrators, the site clinical chief and front-line staff to create an immediate action plan to engage appropriate stakeholders and plan for a rapid response. We took the opportunity to raise questions and concerns related to the emergency department's contingency protocols during site planning sessions, which took place in the emergency department with all staff members who were present at the time. Important changes and decisions were made based on this discussion and quickly led to an action plan that aims to adapt to the number and severity of cases that may occur. We 
discussed possible changes in emergency department structure to optimize infection prevention and control (Figure 1) and potential spaces adjacent to the emergency department that would allow us to isolate patients with respiratory illness from nonrespiratory cases.

We decided to establish a respiratory centre by reassigning office space that was occupied by surgery services. We also created a negative pressure room in the emergency department's trauma bay to establish a space separate from other areas in the emergency department where staff providing care inside the space would be equipped with full PPE.

We developed a flexibility and adaptability agenda by identifying physicians with low workloads because of the closure of less urgent health services. The agenda was developed to address the loss of support owing to travel and work restrictions for locums and providers who work at more than 1 site. This approach was also applied to registered nurses, licensed practical nurses, medical service aids and cleaners to ensure continuity of service in the event that temporary staff replacements are needed because of quarantine protocols.

We also reviewed existing channels of communication and information sharing to decide which method (e.g., emergency department bulletin board, phone, email lists and text) was fastest and most reliable to inform emergency department staff in our hospital of the most recent guidelines and administrative decisions, and the best available evidence for those decisions. We found that posting updates to the emergency department bulletin board and engaging with other staff and clinicians through mailing lists was the fastest and most effective way of keeping all team members informed. We also explored how best to use the SurgeCon platform to assist in addressing patient surge and to facilitate front-line and administrative communication. ${ }^{4}$

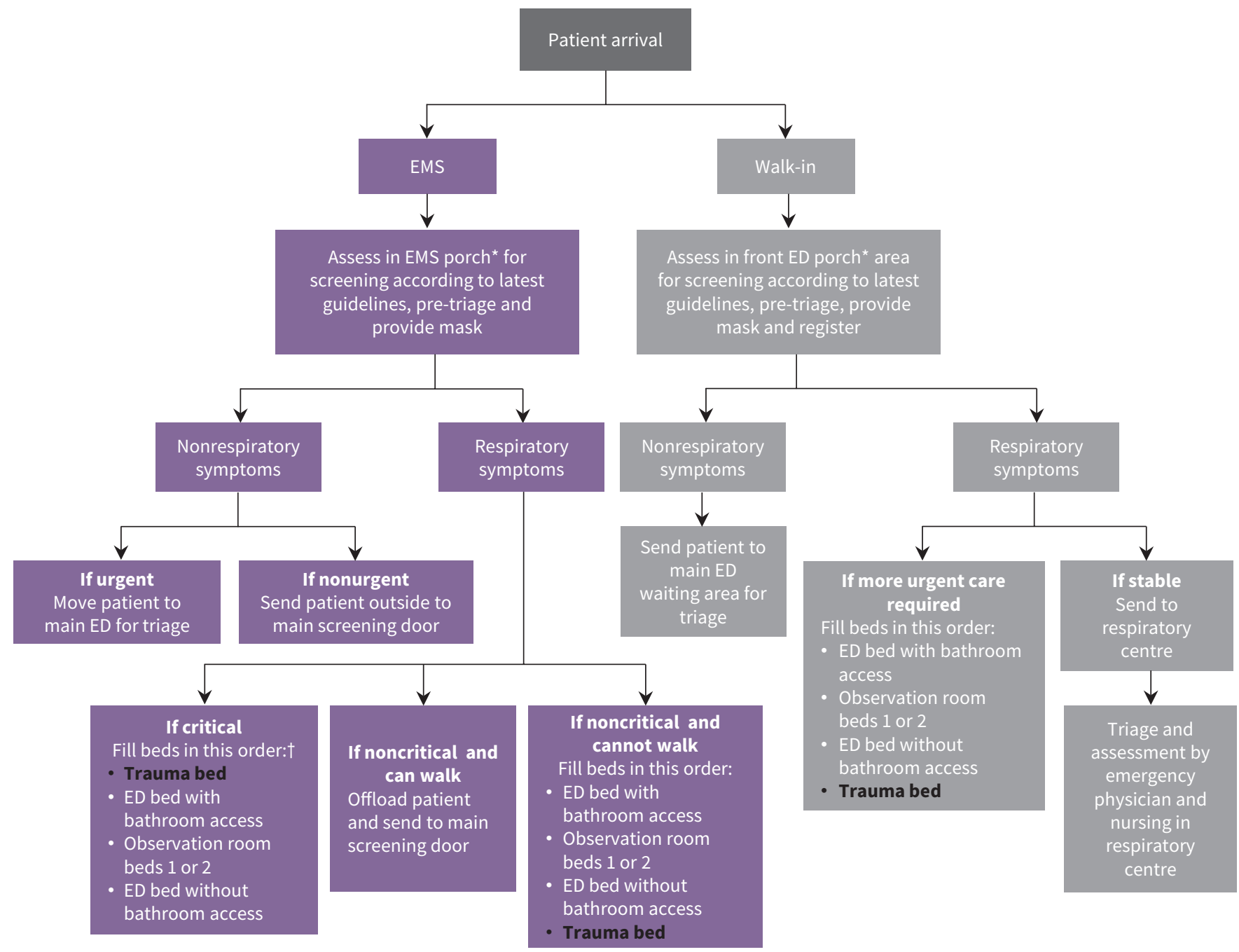

Figure 1: Coronavirus disease 2019 (COVID-19) pandemic flow map for all patients presenting to the emergency department (ED) in Carbonear, NL. The bolded black font indicates a negative pressure room was created in the existing trauma room with a walled-off doffing and donning containment entry. " "EMS porch" and "ED porch" refer to the areas between 2 sets of doors at the ambulance entrance and walk-in entrance. †EMS critical patient bed order prioritizes large rooms for stretcher offload and bathroom availability. Note: EMS = emergency medical services. 
The SurgeCon platform is a locally developed eHealth system that manages emergency department surge by actively tracking wait times based on patient acuity, patient demand (e.g., number of patients not yet seen by a physician and number of patients needing triage) and available resources (e.g., number of emergency department beds occupied, inpatient bed availability and number of admitted patients awaiting transfer). ${ }^{4}$ The platform includes a front-line protocol, which provides tasks (generated via data-processing algorithms) for emergency department staff to complete to help reduce wait times and improve patient flow. ${ }^{4}$

Our emergency department action plan was operationalized within a 36-hour period. We continue to monitor current and forecasted SARS-CoV-2 infections included in NL government reports and emergency department performance data to determine whether we need to make any adjustments to adapt to an ever-evolving situation. We have mobilized administrative and other clinical support based on provincial COVID-19 forecasting and emergency department performance data (e.g., establishment of a hospital emergency operations centre, more frequent "Lean" meetings for front-line staff, and increasing housekeeping and maintenance staff required for redesign and sanitation).

We discussed preventive safety measures staff need to take to reduce the likelihood of transmission inside and outside the hospital. The segregation of patients through the creation of designated areas in the emergency department with physical barriers allowed for a reduction in the usage of PPE required for aerosolgenerating procedures. The type of PPE to be worn in each of the designated areas was assigned based on guidance from the Newfoundland and Labrador Eastern Regional Health Authority. ${ }^{5}$

Being fully aware of the psychological well-being of staff, we have arranged for regular teleconference meetings to address all existing and arising needs. Moreover, we prepared staff members to expect a heightened level of social isolation, and to expect that they may cause anxiety for members of the public when outside the emergency department given that they are more likely to be recognized as front-line providers because of the lack of anonymity associated with rural and remote locations. We have provided staff members with the contact information for Eastern Health's rapid response team, which can provide mental health, technical and clinical support through rapid assessments or site visits. We also shared information on how to access mental health resources developed specifically for health care workers. ${ }^{6}$

The emergency department will likely be the first point of contact for many patients infected with SARS-CoV-2. We need to continue working within the framework of a regional response plan, and planning for self-reliance will be critical if a patient surge occurs. The measures taken proactively by the Carbonear emergency department to allow us to effectively respond to a potential increase in patients needing emergency care, while ensuring patient and staff safety, may be of use to others working in rural hospitals.

\section{References}

1. Working Party of the Australasian College for Emergency Medicine. Managing COVID-19 across the Info-Pacific: a guide to emergency departments with limited resources. Melbourne (AU): Australasian College for Emergency Medicine; 2020:763.

2. Operational considerations for case management of COVID-19 in health facility and community: interim guidance. Geneva: World Health Organization; 2020. Available: https://apps.who.int/iris/bitstream/handle/10665/331492/WHO -2019-nCoV-HCF_operations-2020.1-eng.pdf (accessed 2020 Mar. 20).

3. Clinical management of patients with moderate to severe COVID-19 - interim guidance. Ottawa: Government of Canada; 2020. Available: www.canada.ca/ en/public-health/services/diseases/2019-novel-coronavirus-infection/clinical -management-covid-19.html (accessed 2020 Apr. 7).

4. Patey C, Norman P, Araee M, et al. SurgeCon: priming a community emergency department for patient flow management. West J Emerg Med 2019;20:654-65.

5. New procedural mask requirements for staff, physicians and residents at Eastern Health. St. John's (NL): NL Eastern Regional Health Authority; 2020:1-3.

6. Mental health and COVID-19: resources for health care workers. Toronto: Centre for Addiction and Mental Health; 2020. Available: https://www.camh.ca/en/ health-info/mental-health-and-covid-19/information-for-professionals (accessed 2020 Apr. 7).
Competing interests: Christopher Patey and Paul Norman are the cofounders of SurgeCon Innovations. Christopher Patey and Shabnam Asghari are principal investigators, Oliver Hurley is a research coordinator and Paul Norman is a collaborator on a project entitled "SurgeCon: An Emergency Department Surge Management Platform," funded by the Canadian Institutes of Health Research (CIHR). Paul Norman has a royalty sharing agreement with MOBIA Technology Innovations and the Newfoundland and Labrador Eastern Regional Health Authority.

This article has been peer reviewed.

Affiliations: Discipline of Family Medicine (Patey); Discipline of Family Medicine, Centre for Rural Health Studies (Asghari, Hurley), Faculty of Medicine, Memorial University of Newfoundland, St. John's, NL; Eastern Health, Carbonear Institute for Rural Reach and Innovation by the Sea (Patey, Norman), Carbonear General Hospital, Carbonear, NL
Contributors: Paul Norman and Christopher Patey conceived of the presented idea. Shabnam Asghari and Oliver Hurley developed the theoretical framework and designed the manuscript. All authors drafted the manuscript, reviewed and revised it critically or made important intellectual contributions to content, gave final approval of the version to be published and agreed to be accountable for all aspects of the work.

Funding: The authors are currently receiving financial support from the Canadian Institutes of Health Research (CIHR), the Government of Newfoundland and Labrador, Eastern Health, Memorial University of Newfoundland, and the Trinity Conception Placentia Health Foundation for the continued development of the SurgeCon platform to improve emergency department wait times and patient satisfaction.

Acknowledgement: The authors acknowledge all front-line providers and administrators of the Carbonear emergency department.

Correspondence to: Christopher Patey, christopher.patey@med.mun.ca 